\title{
Estimando las participaciones de mercado de largo plazo en el servicio móvil peruano: Una aplicación de las Cadenas de Markov
}

\author{
Manuel Gavilano Aspillaga ${ }^{1}$ y Nikolai Tovar Soto ${ }^{2}$
}

Resumen: Este breve documento muestra de manera directa y sencilla la estimación de las participaciones de mercado que tendrían las empresas operadoras móviles en el largo plazo, entendiéndose como largo plazo aquella situación en que las participaciones de mercado oscilan en muy pequeñas variaciones. Para cumplir con este objetivo se emplean las denominadas Cadenas de Markov, donde las Matrices de Transición se forman a través de los estados de migraciones de una empresa operadora hacia otra mediante el proceso de Portabilidad Numérica Móvil (PNM). Las conclusiones del trabajo señalan que, en el largo plazo Telefónica del Perú tendría la mayor participación de mercado (36.4\%), seguido de América Móvil (35\%); y finalmente, Entel $(17.4 \%)$ y Viettel $(11.2 \%)$.

Palabras claves: Cadenas de Markov; participaciones de mercado; telefonía móvil.

\section{Estimating the long-term market shares in the Peruvian mobile telephony service: An application of the Markov Chains}

\begin{abstract}
This brief document shows in a simple and direct way the estimation of the market shares that mobile operators will have in the long term, understanding as a long term that situation in which the market shares oscillate in very small variations. To achieve this goal, the so-called Markov Chains are used, where the Transition Matrices are formed through the states of migration between companies using the Mobile Number Portability (PNM) process. The conclusions of this paper indicate that in the long term Telefónica del Perú will have the largest market share (36.4\%), followed by América Móvil, (35\%); and finally Entel (17.4\%) and Viettel $(11.2 \%)$.
\end{abstract}

Keywords: Markov Chains; market shares; mobile telephony.

Recibido: 18/01/2019. Aceptado: 18/04/2019. Publicado online: 13/05/2019.

\footnotetext{
CLos autores. Este artículo es publicado por la Revista PESQUIMAT de la Facultad de Ciencias Matemáticas, Universidad Nacional Mayor de San Marcos. Este es un artículo de acceso abierto, distribuido bajo los términos de la licencia Creative Commons Atribucion-No yor de San Marcos. Este es un artículo de acceso abierto, distribuido bajo los términos de la licencia Creative Commons Atribucion-No ción y reproducción en cualquier medio, siempre que la obra original sea debidamente citada. Para información, por favor póngase en contacto con revistapesquimat.matematica@unmsm.edu.pe
}

\footnotetext{
${ }^{1}$ UNMSM, Facultad de Ciencias Matemáticas, e-mail: manuel.gavilano@unmsm.edu.pe

${ }^{2}$ UNCP, Facultad de Economía, e-mail: nikolaieliastovar@gmail.com
} 


\section{Introducción}

Una Cadena de Markov es un proceso estocástico que satisface la propiedad Markoviana, la cual establece que el pasado y el futuro son independientes cuando se conoce el presente. Esto es, si se conoce el estado presente de un proceso, la información adicional del proceso en el pasado no es útil para realizar la mejor predicción de su futuro. (Esto se detalla muy brevemente en la sección 3 de este documento).

En la actualidad, las Cadenas de Markov vienen siendo utilizadas ampliamente en distintos campos de las ciencias. En este documento, se emplean dos versiones de Cadenas de Markov para cumplir con los siguientes propósitos en el mercado de la telefonía móvil ${ }^{1}$ :

- Cadenas de Markov Homogénea, las cuales permiten estimar las cuotas de mercado que las empresas operadoras tendrían en el largo plazo, considerando las condiciones actuales (por ejemplo, la migración entre empresas operadoras producto de la $\mathrm{PNM}^{2}$ ).

- Cadenas de Markov Variante en el tiempo, las cuales permiten realizar predicciones de las cuotas de mercado que las empresas operadoras tendrían en el corto plazo.

Es importante resaltar que los objetivos mencionados ya han sido aplicados en mercados de telecomunicaciones de otros países, por lo que el objetivo del documento es aplicarlo al caso peruano. Así, Ching [1] utiliza Cadenas de Markov para pronosticar las participaciones de mercado a partir de datos simulados; Gomewa et al. [4] analizaron el comportamiento dinámico de la PNM por parte de los usuarios del mercado móvil en Nigeria, y estiman las participaciones de mercado de largo plazo; por su parte, Mendoza et al. [5] realizaron un estudio similar para el caso del mercado móvil mexicano.

A diferencia de otras técnicas que predicen a partir de sus propios valores pasados, las Cadenas de Markov son relevantes toda vez que considera tanto información presente como las interrelaciones que pueda existir con otras series. De este modo, las Cadenas de Markov son sistemáticas (debido a que abordan todos los flujos posibles entre estados a través de las Matrices de Transición) y no se limita en las estructuras causales del sistema, por lo que no es necesario recurrir a métodos econométricos tradicionales.

\section{Estado actual del servicio móvil peruano}

El servicio móvil contempla constantes cambios como producto del incremento de la intensidad competitiva que ejercen las empresas operadoras (quienes ofrecen servicios con mejores atributos y mayor calidad). En el caso peruano, este comportamiento agresivo de las empresas operadoras ha llevado a que frecuentemente compitan por atraer a usuarios de la competencia y a fidelizar a los suyos. Como resultado, este comportamiento ha llevado a que los usuarios respondan ante las constantes mejoras e incentivos para aquellos que migren entre empresas operadoras (por ejemplo: duplicar los GB asignados en el plan durante 6-12 meses, reducción de precios de equipos).

El análisis se centra en las cuatro operadoras más grandes, dado que en su conjunto cubren el $99 \%$ de las líneas en servicio. A saber, Telefónica, América Móvil, Entel y Viettel ${ }^{3}$. Si bien, hasta hace algunos años, Telefónica y América Móvil mantenían conjuntamente más del $95 \%$ de las líneas móviles en servicio; es con la entrada de nuevas empresas operadoras, el relanzamiento

\footnotetext{
${ }^{1}$ Las Cadenas de Markov son fácilmente aplicadas a cualquier mercado de telecomunicaciones. Asimismo, las predicciones realizadas en esta investigación supone que las condiciones actuales se mantienen en el tiempo.

${ }^{2}$ PNM: Portabilidad Numérica Móvil.

${ }^{3}$ No se considera a Incacel por tener una muy baja participación de mercado en comparación al resto empresas.
} 
de la PNM y el desbloqueo de equipos terminales que la intensidad competitiva por parte de las empresas operadoras se incrementó rápidamente (vía una reducción de los costos de traspaso). Esto se observa claramente en la evolución de las participaciones de mercado (Ver Figura 1).

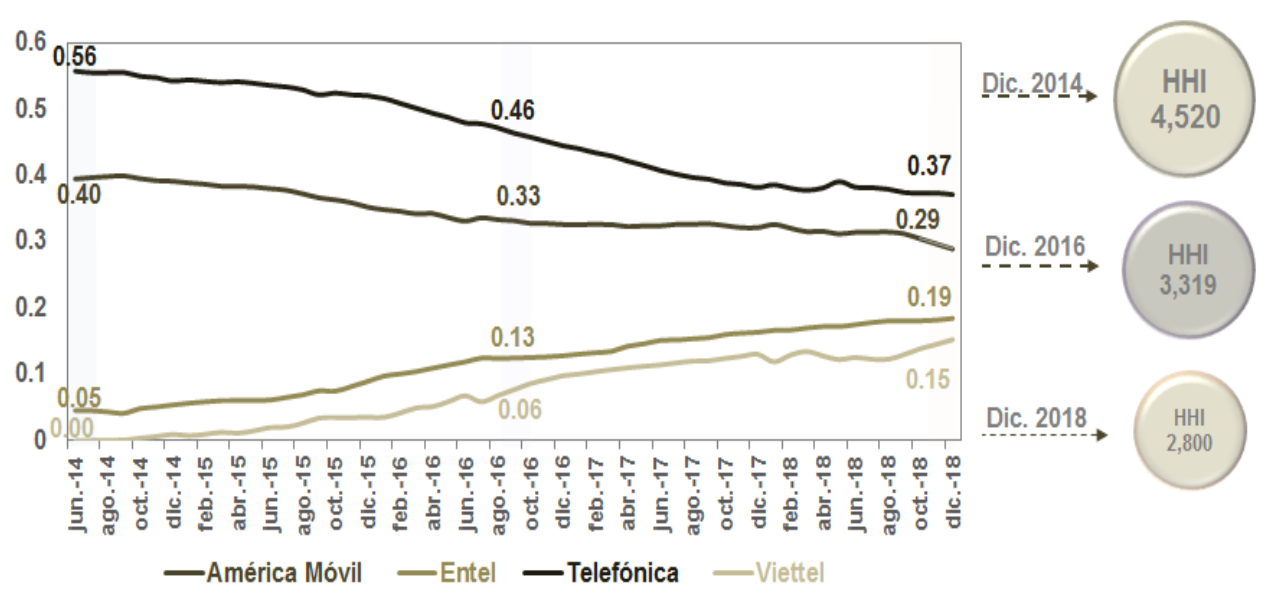

Figura 1. Dinamismo de cuotas de mercado e Índice de Concentración (HHI) Fuente: OSIPTEL.

De esta manera, en la figura anterior se observa la reducción de las brechas de participaciones de mercado entre las empresas operadoras (y con ello una reducción del Índice de HirshmanHerfindahl (HHI)), lo que muestra un mercado menos concentrado (el HHI se redujo de 4,520 a 2,800 entre diciembre de 2014 y diciembre de 2018). Dichos cambios en la evolución de la participación de mercado se deben principalmente al movimiento de usuarios entre empresas operadoras, más que al ingreso de nuevos usuarios.

Este aumento de las participaciones de mercado de las empresas operadoras más pequeñas (principalmente Entel) a costa de las empresas más grandes se muestra en el siguiente figura. Es en base a dichas migraciones o traspasos que se estiman las participaciones de mercado de largo plazo. (Ver Figura 2).

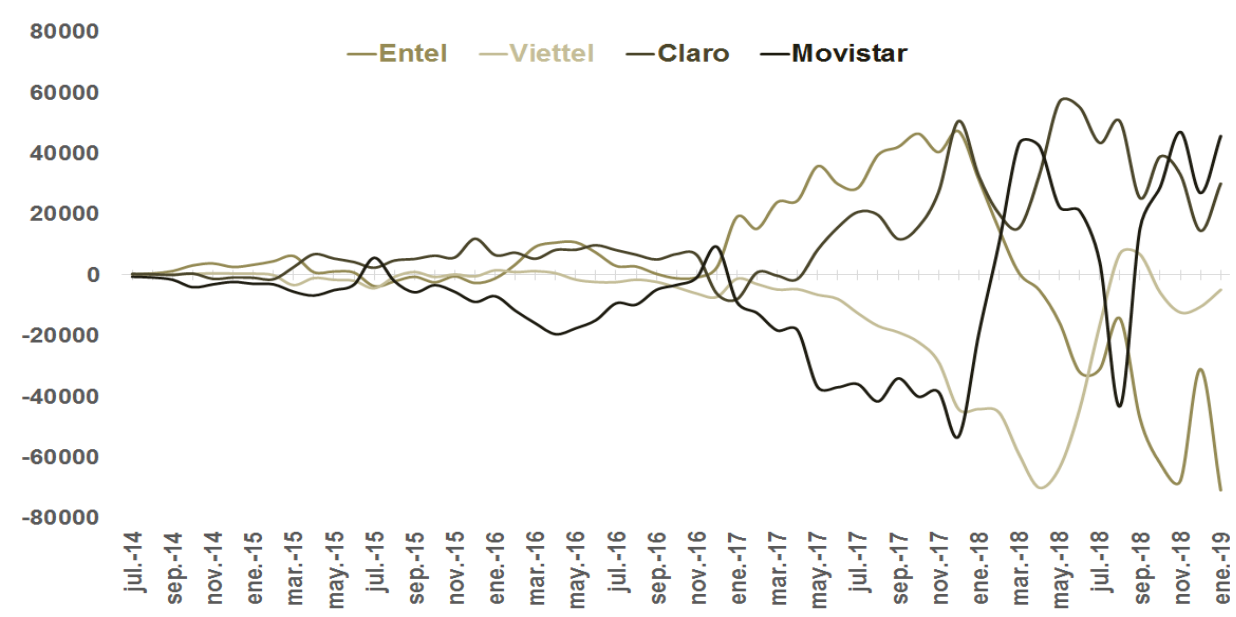

Figura 2. Dinamismo en posición neta de usuarios en PNM por empresa Fuente: OSIPTEL. 


\section{Marco Teórico}

Si bien existen diversas versiones de Cadenas de Markov para predecir valores futuros o para estimar participaciones de mercado de largo plazo, es necesario resaltar que estas deben ser aplicadas dependiendo del contexto por el que atraviesa el mercado analizado. La importancia del empleo de Cadenas de Markov -frente a otras metodologías- recae en que este es un proceso sistemático y utiliza información presente de distintos estados.

En este documento se emplea la versión de las Cadenas de Markov más utilizada para estimar las participaciones de mercado de largo plazo (versión homogénea) y la versión de Cadenas de Markov para estimar las participaciones en el corto plazo (versión variante en el tiempo).

\subsection{Modelo de Cadenas de Markov Homogénea}

Una Cadena de Markov se compone del vector de condiciones iniciales y de la matriz de transición, donde se define los movimientos de las personas dentro de un conjunto finito de estados $s_{i}$.

$$
s \in S, S=\left\{s_{1}, s_{2}, s_{3}, \ldots, s_{M-1}, s_{M}\right\}, M<\infty
$$

Para los propósitos del presente trabajo $S$ tiene 4 componentes, representando a las cuatro empresas operadores móviles más importantes del mercado peruano: Telefónica, América Móvil, Entel y Viettel.

Los movimientos de las personas se dan a través de puntos discretos en el tiempo, donde la probabilidad de estar en el tiempo $t$ sólo va a depender del tiempo inmediato anterior, es decir:

$$
P\left\{X_{t+1}=j \mid X_{t}=i, X_{t-1}=i_{t-1}, \ldots, X_{1}=i_{1}\right\}=P\left\{X_{t+1}=j \mid X_{t}=i\right\} ; \forall t
$$

Se puede ver claramente que es un proceso sin memoria o Cadena de Markov de primer orden. Por otro lado, se dice que es homogénea porque las probabilidades de transición son invariantes en el tiempo,

$$
P\left\{X_{t+1}=j \mid X_{t}=i\right\}=p_{i, j}
$$

La probabilidad $p_{i, j}$ representa la posibilidad de que un usuario de determinada empresa operadora $i$ migre a $j$ en el siguiente periodo. Luego, las distintas probabilidades de transición entre diferentes empresas operadoras, se pueden agrupar en una matriz cuadrada de orden de 4 , la cual es llamada Matriz de Transición ${ }^{4}$.

$$
\mathbf{P}=\left(\begin{array}{llll}
p_{1,1} & p_{1,2} & p_{1,3} & p_{1,4} \\
p_{2,1} & p_{2,2} & p_{2,3} & p_{2,4} \\
p_{3,1} & p_{3,2} & p_{3,3} & p_{3,4} \\
p_{4,1} & p_{4,2} & p_{4,3} & p_{4,4}
\end{array}\right)
$$

La propiedad que debe cumplir la Matriz de Transición es que el conjunto de estados deben ser mutuamente excluyentes, por lo que la suma de probabilidades (por fila o columna) siempre debe ser igual a 1. El caso presentado es de una Matriz de Transición por filas.

El vector de condiciones iniciales es estimado sobre la base de datos históricos, el cual se define por $\mathbf{Y}_{1}$. Dado que se trata de participaciones de mercado, se debe cumplir -por el axioma de completitud- que la suma de componentes de este vector sea 1.

$$
\mathbf{Y}_{1}=\left(\begin{array}{llll}
y_{1} & y_{2} & y_{3} & y_{4}
\end{array}\right)
$$

\footnotetext{
${ }^{4}$ En adelante, todas las expresiones matemáticas en negrita representarán matrices.
} 
Una vez obtenidos los insumos mencionados, la predicción de las participaciones de mercado para el periodo siguiente se obtiene de la siguiente manera.

$$
\mathbf{Y}_{t+1}=\mathbf{Y}_{t} \times \mathbf{P}
$$

Empleando esta ecuación de manera recursiva conjuntamente con el vector de condiciones iniciales, se obtiene la predicción para cualquier punto deseado en el tiempo:

$$
\mathbf{Y}_{t+1}=\mathbf{Y}_{1} \times \mathbf{P}^{t}
$$

Bajo el supuesto de homogeneidad de la matriz de transición se obtiene las participaciones de mercado de largo plazo o estacionarias. Siendo $\mathbf{Q}$ el vector de participaciones de mercado de largo plazo de cada empresa operadora, esta debe cumplir lo siguiente:

$$
\mathbf{Q} \times \mathbf{P}=\mathbf{Q} \quad \text { sujeto a: } \quad \sum_{i=1}^{4} q_{i}=1,
$$

donde $q_{i}$ son los elementos del vector de largo plazo.

\subsection{Modelo de Cadenas de Markov Variante en el tiempo}

Una restricción al modelo anterior es la homogeneidad de la Matriz de Transición, el cual es un supuesto que probablemente no siempre se cumpla, por lo que una aproximación alternativa sería que la Matriz de Transición fuese variante en el tiempo. Para proceder bajo este criterio se necesita obtener dos Matrices de Transición, las cuales permitan estimar la variación que existe entre dos periodos de tiempos contiguos $(\Delta P))$, y luego generalizar dicha variación para el resto de periodos.

De esta manera, las predicciones quedarían de la siguiente forma:

$$
\begin{aligned}
& \mathbf{Y}_{2}= \mathbf{Y}_{\mathbf{1}} \times \mathbf{P}_{1} \\
& \mathbf{Y}_{3}=\mathbf{Y}_{1} \times \mathbf{P}_{1} \times \mathbf{P}_{2} \\
& \vdots \\
& \mathbf{Y}_{t+1}=\mathbf{Y}_{1} \times \mathbf{P}_{1} \times \mathbf{P}_{2} \ldots \mathbf{P}_{t}=\mathbf{Y}_{1} \prod_{n=1}^{t} \mathbf{P}_{n}
\end{aligned}
$$

Nótese que ahora existen " $n$ " Matrices de Transición, además:

$$
\mathbf{P}_{t+1}=\mathbf{P}_{t}+\Delta \mathbf{P}
$$

el cual permitirá estimar la variabilidad entre dos Matrices de Transición contiguas, permitiendo que dichas matrices varíen con el tiempo (levantando el supuesto de homogeneidad).

\section{Aplicación práctica de las Cadenas de Markov}

Como se mencionó anteriormente, las Cadenas de Markov Homogéneas frecuentemente son empleadas para estimar participaciones de mercado que tendrían los operadores en el largo plazo o en un estado de convergencia. Mientras que el resto de versiones existentes de Cadenas de Markov (que son variantes en el tiempo) permiten predecir las participaciones de mercado para los periodos siguientes al analizado. 


\subsection{Aplicación para Cadenas de Markov Homogénea}

Con el propósito de estimar las participaciones de mercado de largo plazo (estacionarias o convergentes), se requiere obtener la Matriz de Transición a partir de los datos de líneas móviles en servicio y la posición neta agregada de las empresas operadoras producto de las migraciones realizadas mediante PNM (ver Tabla 1).

Tabla 1. Líneas en servicio que realizaron Portabilidad Numérica Móvil en el periodo (Marzo-Noviembre 2018)

\begin{tabular}{|c|cccc|c|}
\hline \hline $\begin{array}{c}\text { Marzo-Noviembre } \\
\text { (Año 2018) }\end{array}$ & Entel & América Móvil & Telefónica & Viettel & Total \\
\hline \hline Entel & $6,550,592$ & 836,004 & 665,866 & 197,457 & $8,249,919$ \\
América Móvil & 734,729 & $12,572,199$ & 770,232 & 280,563 & $14,357,723$ \\
Telefónica & 660,770 & 894,936 & $14,928,872$ & 297,064 & $16,781,642$ \\
Viettel & 194,340 & 347,492 & 397,337 & $5,074,673$ & $6,013,842$ \\
\hline \hline Total & $8,140,431$ & $14,650,631$ & $16,762,307$ & $5,849,757$ & $45,403,126$ \\
\hline \hline $\begin{array}{c}\text { Cuotas de mercado } \\
\text { (inicial) }\end{array}$ & $\mathbf{0 . 1 7 9 3}$ & $\mathbf{0 . 3 2 2 7}$ & $\mathbf{0 . 3 6 9 2}$ & $\mathbf{0 . 1 2 8 8}$ & $\mathbf{1 . 0}$ \\
\hline \hline
\end{tabular}

Fuente: OSIPTEL.

Los elementos que aparecen sombreados en la Tabla $1\left(a_{i i}\right)$ representan la cantidad de líneas móviles en servicio que tenía cada empresa operadora al final del periodo anterior (para este caso, en febrero de 2018). El resto de valores registrados en la Tabla 1 son los movimientos agregados de la migración de las líneas móviles en servicio entre las distintas empresas operadoras, de tal modo que el elemento $\left[a_{1,2}=(836,004)\right]$ (en fila 1 -columna 2 ) se interpreta como el número de líneas móviles que migraron de Entel hacia América Móvil en el transcurso de los meses señalados. Mientras que la última fila de la tabla muestra las participaciones de mercado iniciales de cada empresa operadora.

$$
\mathbf{Y}_{1}=\left(\begin{array}{llll}
0.1793 & 0.3227 & 0.3692 & 0.1288
\end{array}\right)
$$

A partir de la tabla anterior se obtiene la Matriz de Transición por filas (ver Tabla 2). El procedimiento a seguir es convertir las frecuencias absolutas en frecuencias relativas. Por ejemplo, el elemento $p_{1,2}$ puede ser estimado mediante el siguiente ratio:

$$
p_{1,2}=\frac{836,004}{8,249,919}=0.101335
$$

Si se aplica la misma operación para el resto de elementos se completa la Matriz de Transición $\mathbf{P}$ (Tabla 2), la misma que debe cumplir con los requisitos ya señalados.

Tabla 2. Matriz de Probabilidades de Transición en el periodo corriente (" $t$ ": Marzo-Noviembre 2018)

\begin{tabular}{|c|cccc|}
\hline \hline $\begin{array}{c}\text { Marzo-Noviembre } \\
\text { (Año 2018) }\end{array}$ & Entel & América Móvil & Telefónica & Viettel \\
\hline \hline Entel & 0.794019 & 0.101335 & 0.080712 & 0.023934 \\
América Móvil & 0.051173 & 0.875640 & 0.053646 & 0.019541 \\
Telefónica & 0.039375 & 0.053328 & 0.889595 & 0.017702 \\
Viettel & 0.032315 & 0.057782 & 0.066070 & 0.843832 \\
\hline \hline
\end{tabular}

Fuente: Elaboración propia. 
Luego, para obtener las participaciones de mercado de largo plazo se necesita un vector $(\mathbf{Q})$ de dimensión $1 \times 4$.

$$
\mathbf{Q}=\left(\begin{array}{llll}
q_{1} & q_{2} & q_{3} & q_{4}
\end{array}\right)
$$

Dado que el orden en las operaciones de matrices es relevante, continuando con la lógica en la enumeración, $q_{1}, q_{2}, q_{3}$ y $q_{4}$ corresponden a las participaciones de mercado de largo plazo de Entel, América Móvil, Telefónica y Viettel, respectivamente. Para proceder con la estimación respectiva, se efectua algunas operaciones algebraicas a la ecuación 2 . De este modo, aplicando transpuesta a ambos lados de la igualdad se tiene que:

$$
\begin{aligned}
\mathbf{Q} \times \mathbf{P} & =\mathbf{Q} \\
(\mathbf{Q} \times \mathbf{P})^{\prime} & =\mathbf{Q}^{\prime} \\
\mathbf{P}^{\prime} \times \mathbf{Q}^{\prime} & =\mathbf{Q}^{\prime} .
\end{aligned}
$$

Luego, reemplazando los respectivos valores:

$$
\begin{gathered}
{\left[\begin{array}{llll}
0.7940 & 0.0512 & 0.0394 & 0.0323 \\
0.1013 & 0.8756 & 0.0533 & 0.0578 \\
0.0807 & 0.0536 & 0.8896 & 0.0661 \\
0.0239 & 0.0195 & 0.0177 & 0.8438
\end{array}\right] \times\left[\begin{array}{l}
q_{1} \\
q_{2} \\
q_{3} \\
q_{4}
\end{array}\right]=\left[\begin{array}{l}
q_{1} \\
q_{2} \\
q_{3} \\
q_{4}
\end{array}\right]} \\
{\left[\begin{array}{l}
0.7940 q_{1}+0.0512 q_{2}+0.0394 q_{3}+0.0323 q_{4} \\
0.1013 q_{1}+0.8756 q_{2}+0.0533 q_{3}+0.0578 q_{4} \\
0.0807 q_{1}+0.0536 q_{2}+0.8896 q_{3}+0.0661 q_{4} \\
0.0239 q_{1}+0.0195 q_{2}+0.0177 q_{3}+0.8438 q_{4}
\end{array}\right]=\left[\begin{array}{l}
q_{1} \\
q_{2} \\
q_{3} \\
q_{4}
\end{array}\right]}
\end{gathered}
$$

Además, se tiene la siguiente restricción de completitud:

$$
q_{1}+q_{2}+q_{3}+q_{4}=1,
$$

de donde se obtiene las siguientes equivalencias:

$$
\begin{aligned}
& q_{1}=1-q_{2}-q_{3}-q_{4} \\
& q_{2}=1-q_{1}-q_{3}-q_{4} \\
& q_{3}=1-q_{1}-q_{2}-q_{4} \\
& q_{4}=1-q_{1}-q_{2}-q_{3}
\end{aligned}
$$

Reemplazando (6) en (5) se obtiene lo siguiente:

$$
\begin{gathered}
{\left[\begin{array}{l}
0.7940 q_{1}+0.0512 q_{2}+0.0394 q_{3}+0.0323 q_{4} \\
0.1013 q_{1}+0.8756 q_{2}+0.0533 q_{3}+0.0578 q_{4} \\
0.0807 q_{1}+0.0536 q_{2}+0.8896 q_{3}+0.0661 q_{4} \\
0.0239 q_{1}+0.0195 q_{2}+0.0177 q_{3}+0.8438 q_{4}
\end{array}\right]=\left[\begin{array}{l}
1-q_{2}-q_{3}-q_{4} \\
1-q_{1}-q_{3}-q_{4} \\
1-q_{1}-q_{2}-q_{4} \\
1-q_{1}-q_{2}-q_{3}
\end{array}\right]} \\
{\left[\begin{array}{l}
0.7940 q_{1}+1.0512 q_{2}+1.0394 q_{3}+1.0323 q_{4} \\
1.1013 q_{1}+0.8756 q_{2}+1.0533 q_{3}+1.0578 q_{4} \\
1.0807 q_{1}+1.0536 q_{2}+0.8896 q_{3}+1.0661 q_{4} \\
1.0239 q_{1}+1.0195 q_{2}+1.0177 q_{3}+0.8438 q_{4}
\end{array}\right]=\left[\begin{array}{l}
1 \\
1 \\
1 \\
1
\end{array}\right]}
\end{gathered}
$$

Una manera alternativa y sencilla de solución es presentar este último resultado en forma de producto de matrices. 


$$
\begin{gathered}
{\left[\begin{array}{rrrr}
0.7940 & 1.0512 & 1.0394 & 1.0323 \\
1.1013 & 0.8756 & 1.0533 & 1.0578 \\
1.0807 & 1.0536 & 0.8896 & 1.0661 \\
1.0239 & 1.0195 & 1.0177 & 0.8438
\end{array}\right] \times\left[\begin{array}{l}
q_{1} \\
q_{2} \\
q_{3} \\
q_{4}
\end{array}\right]=\left[\begin{array}{l}
1 \\
1 \\
1 \\
1
\end{array}\right]} \\
\mathbf{A}_{4 x 4} \times \mathbf{Q}_{4 X 1}=\mathbf{1}_{4 x 1}
\end{gathered}
$$

Premultiplicando, a ambos lados de la ecuación, por la matriz inversa de $\mathbf{A}\left(\mathbf{A}^{-\mathbf{1}}\right)$, se tiene que:

$$
\begin{aligned}
\mathbf{A}_{4 x 4}^{-1} \times \mathbf{A}_{4 x 4} \times \mathbf{Q}_{4 x 1} & =\mathbf{A}^{-1} \times \mathbf{1}_{4 x 1} \\
\mathbf{Q}_{4 x 1} & =\mathbf{A}_{4 x 4}^{-1} \times \mathbf{1}_{4 x 1},
\end{aligned}
$$

donde $\mathbf{Q}_{4 x 1}$ es simplemente el producto matricial de la inversa de $\mathbf{A}_{4 x 4}\left(\mathbf{A}_{4 x 4}^{-1}\right)$ y el vector $\mathbf{1}_{4 x 1}{ }^{5}$. Reemplazando por sus valores respectivos se obtiene que:

$$
\left[\begin{array}{l}
q_{1} \\
q_{2} \\
q_{3} \\
q_{4}
\end{array}\right]=\left[\begin{array}{cccc}
-3.0222 & 0.8305 & 1.1027 & 1.2632 \\
0.6996 & -3.8851 & 1.8181 & 1.7174 \\
1.2102 & 1.8527 & -4.1928 & 1.4941 \\
1.3624 & 1.4520 & 1.5221 & -4.2247
\end{array}\right] \times\left[\begin{array}{c}
1 \\
1 \\
1 \\
1
\end{array}\right]
$$

El desarrollo de esta última expresión permite obtener las participaciones de mercado de largo plazo:

$$
\mathbf{Q}=\left[\begin{array}{l}
q_{1} \\
q_{2} \\
q_{3} \\
q_{4}
\end{array}\right]=\left[\begin{array}{l}
0.174 \\
0.350 \\
0.364 \\
0.112
\end{array}\right]
$$

De este modo, se obtiene la participación de mercado de largo plazo para las cuatro operadoras más importantes del mercado móvil peruano. En efecto, la empresa operadora con mayor participación sería Telefónica con un 36.4\%; seguido muy de cerca por América Móvil con $35.0 \%$. Más lejanamente, se encontrarían Entel y Viettel con participaciones de $17.4 \%$ y $11.2 \%$, respectivamente.

\subsection{Aplicación para Cadenas de Markov Variante en el tiempo}

La aplicación de esta versión de las Cadenas de Markov requiere de al menos dos Matrices de Transición de dos puntos contiguos en el tiempo. Dado que ya se cuenta con una Matriz de Transición para el periodo de Marzo a Noviembre de 2018, se construye una matriz adicional correspondiente al periodo anterior (Junio del 2017 a Febrero del 2018), el resultado se presenta en la tabla a continuación:

\footnotetext{
${ }^{5}$ Esta multiplicación es posible ya que cumplen con las condiciones de dimensión
} 
Tabla 3. Matriz de Probabilidades de Transición en el periodo anterior (" $t$-1": Junio 2017 - Febrero 2018)

\begin{tabular}{|c|cccc|}
\hline \hline $\begin{array}{c}\text { Junio-Febrero } \\
(\text { Año 2017/2018) }\end{array}$ & Entel & América Móvil & Telefónica & Viettel \\
\hline \hline Entel & 0.888184 & 0.055699 & 0.048533 & 0.007584 \\
América Móvil & 0.036072 & 0.934446 & 0.024611 & 0.004872 \\
Telefónica & 0.034887 & 0.039403 & 0.920793 & 0.004917 \\
Viettel & 0.030044 & 0.035859 & 0.028796 & 0.905300 \\
\hline \hline
\end{tabular}

Fuente. Elaboración propia.

Luego, la sustracción de las dos Matrices de Transición permite encontrar la variación respectiva. Dicha variación se supone constante para el resto de periodos, con lo cual se puede estimar nuevas Matrices de Transición para distintos periodos en adelante. Realizando dicha operación se tiene $\Delta \mathbf{P}$ :

$$
\Delta \mathbf{P}=\left(\begin{array}{cccc}
-0.094165 & 0.045636 & 0.032179 & 0.016351 \\
0.015101 & -0.058805 & 0.029035 & 0.014669 \\
0.004488 & 0.013925 & -0.031198 & 0.012785 \\
0.002271 & 0.021923 & 0.037274 & -0.061468
\end{array}\right)
$$

Dado que se cuenta con dos Matrices de Transición, para los fines del documento se utilizará el promedio de ambas matrices.

Tabla 4. Matriz de Probabilidades de Transición promedio (Promedio(“t, $t$-1"): Junio 2017 - Noviembre 2018)

\begin{tabular}{|c|cccc|}
\hline \hline $\begin{array}{c}\text { Matriz promedio } \\
\left(\mathbf{P}_{1}\right)\end{array}$ & Entel & América Móvil & Telefónica & Viettel \\
\hline \hline Entel & 0.841101 & 0.078517 & 0.064623 & 0.015759 \\
América Móvil & 0.043622 & 0.905043 & 0.039128 & 0.012206 \\
Telefónica & 0.037131 & 0.046366 & 0.905194 & 0.011309 \\
Viettel & 0.031180 & 0.046821 & 0.047433 & 0.874566 \\
\hline \hline
\end{tabular}

Fuente. Elaboración propia.

En base a la Matriz de Transición construida como el promedio $\left(\mathbf{P}_{1}\right)$, el vector de condiciones iniciales $\left(\mathbf{Y}_{1}\right)$ y la ecuación 3 se pueden estimar las participaciones de mercado que tendrían las cuatro empresas operadoras en los siguientes periodos. La Tabla 5 resume los resultados para cuatro periodos hacia adelante.

Tabla 5. Predicción de participaciones de mercado para cuatro periodos adelante

\begin{tabular}{|c|cccc|}
\hline \hline $\boldsymbol{t}$ & Entel & América Móvil & Telefónica & Viettel \\
\hline \hline 1 & 0.1793 & 0.3227 & 0.3692 & 0.1288 \\
2 & 0.1725 & 0.3264 & 0.3729 & 0.1281 \\
3 & 0.1585 & 0.3256 & 0.3839 & 0.1319 \\
4 & 0.1419 & 0.3201 & 0.3992 & 0.1387 \\
$\vdots$ & $\vdots$ & $\vdots$ & $\vdots$ & $\vdots$ \\
$\vdots$ & $\vdots$ & $\vdots$ & $\vdots$ & $\vdots$ \\
\hline \hline
\end{tabular}

Fuente. Elaboración propia. 


\section{Conclusión}

El sector de telecomunicaciones se caracteriza por ser muy dinámico, tanto por la intensidad competitiva que ejercen las empresas operadoras como por la aparición de nuevas tecnologías sobre las que se brindan los servicios. A pesar de este dinamismo, muchas veces se requiere predecir los valores que tendrían algunos indicadores relevantes en periodos posteriores, así como la participación de mercado que las empresas operadoras tendrían en largo plazo.

De este modo, empleando las Cadenas de Markov homogéneas se estimaron las participaciones de mercado de largo plazo: América Móvil (35.0\%), Telefónica del Perú (36.4\%), Entel $(17.4 \%)$ y Viettel (11.2\%). Así, manteniéndose las actuales condiciones de mercado y el dinamismo de la PNM, en el largo plazo aún se muestra una mayor participación por parte de Telefónica del Perú y América Móvil.

Adicionalmente, se emplearon las Cadenas de Markov variantes en el tiempo con la finalidad de predecir las participaciones de mercado en el corto plazo, tomando como información las condiciones actuales que experimenta el mercado.

\section{Referencias bibliográficas}

[1] Ching, K. (2015). Market share modelling and forecasting using Markov Chains and alternative models. Recuperado el 04 de octubre del 2018 de goo.gl/uKaR8T.

[2] Hierro, M. \& Guijarro, M. (2006). Un estudio mediante Cadenas de Markov de la dinámica de los movimientos migratorios interterritoriales en España (1990-2003) desde un planteamiento de estimación dinámico. Recuperado el 05 de octubre del 2018 de goo.gl/Udth9L.

[3] Garrocho, C., Jiménez, E. \& Álvarez, J. (1973). Modelando la migración interestatal de México: cadenas de Markov estáticas versus Cadenas de Markov dinámicas con medias móviles. Recuperado del 15 de octubre del 2018 de goo.gl/Pi9oKt.

[4] Gonewa, A., Godwin, U. \& Ngaage, K. (2012). Dynamic Behavior in Customers' Switching and Market Share Analysis: The Markov Model Perspectives. Recuperado el 15 de octubre del 2018 de https://bit.ly/2PflnA2.

[5] Mendoza, K., Hernández, V. \& Romenge, R. (2012). Tendencias en el mercado de teléfonos móviles en el segmento de estudiantes universitarios en México: una aplicación del modelo de Markov. ITAM. México, D.F. Recuperado el 15 de octubre del 2018 de goo.gl/LUJ3C1.

[6] Tolver, A. (2016). An introduction to Markov Chains. Recuperado el 15 de octubre del 2018 de http://web.math.ku.dk/noter/filer/stoknoter.pdf 